\section{Atmospheric cellular convection}

SIR - The implication ${ }^{1}$ that something might readily be deduced from the aspect ratio (width/vertical depth) of convection clouds observed on Venus, on the supposition that they are cellular in formation, is dubious. Laboratory versions of cellular convection, and most theoretical treatments thereof, are dominated by viscosity and thermal conductivity - that is, by molecular transfer mechanisms.

Clouds in the atmospheres of the Earth and Venus are under no similar influences. They are usually significantly affected by wind shear, which causes the cell size to be related to depth and makes them assume a particular orientation. When the wind is very light and without significant shear, as in the case illustrated in the figure, cells may continue to grow in size for a few days, especially if the heat gain and loss is from the sea and out to space by radiation from the lower and upper surfaces of the cloud and are thus dependent on no mechanical connection.

The depth of the cloud layer undergoes no significant change, and so in this case aspect ratios of 100 or more are achieved. There is no definite upper limit. If conditions are not fairly uniform over an area having a diameter of at least several cell widths, a regular pat-

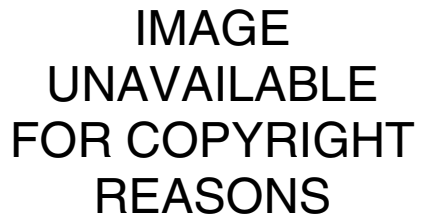

tern is not seen and definitions of cell size become variable and less obvious.

The apparent similarity of pattern in some atmospheric cases to laboratory examples of cellular convection is thus fortuitous; of more interest are the mechanisms of the upward heat flux by which the cloud is as often cooled as it is warmed.

\section{R. S. Scorer}

Department of Mathematics, Imperial College of Science

and Technology,

London SW7 2BZ, UK

BAKER AND SCHUBERT REPLY - We agree with Scorer that the physical mechanisms responsible for convection in atmospheres and in the laboratory must be understood before significance can be attributed to similarities or differences in aspect ratios of convective structures. Precisely for this reason it is appropriate to investigate why the aspect ratios differ in the atmosphere and in the laboratory, or even among different atmospheres.

In the case of the atmosphere of Venus, we suggested a mechanism by which two convective layers interact to form a convective system that spans multiple scale heights and reduces the aspect ratio to typical values found in the Earth's atmosphere ${ }^{1}$. Although the dominant modes of forcing may differ between the two atmospheres (for example, release of latent heat for the Earth versus radiative absorption for Venus), the possibility that both atmospheres may exhibit convection with the same aspect ratio is attractive and should not be considered fortuitous.

\section{R. David Baker II}

Gerald Schubert*

Department of Earth and

Space Sciences,

University of California,

Los Angeles, California 90024, USA

* Also at Institute of Geophysics and Planetary Physics.

1. Baker, R.D. \& Schubert, G. Nature 355, 710-712 (1992)

2. Scorer, R.S. Cloud Investigation by Satellite (picture group 9.26) (Ellis Horwood, Chichester, 1986).

This satellite picture (1246 GMT, 5 March 1982, Nimbus $7,0.70-0.80 \mu \mathrm{m}$, minimum pixel size $0.8 \mathrm{~km}$ ) of convection in a cloud layer about midway between Madeira and the Azores shows the gradual increase in cell size as the air moves slowly westwards and later northwards while the depth of the cloud layer remains almost unchanged and comes to a standstill in a pressure col. about 300 $\mathrm{km}$ from Santa Maria (the most southeasterly point of the Azores, marked S) and the cells become arranged in progressively larger units over possibly two or three days. This case is described in more detail in ref 2. (Copyright University of Dundee.)
Allelic exclusion model questioned

SIR - Kitamura and Rajewsky ${ }^{1}$ have presented evidence that targeted disruption of the membrane exon of the immunoglobulin class $\mu$ heavy chain results in a partial failure of allelic exclusion; that is, they have found another case of allelic inclusion ${ }^{2,3}$. Despite a rather poor agreement between their data and calculations, the authors claim that the 'feedback' of the membrane exon of the $\mu$ chain can account for allelic exclusion at the immunoglogulin $\mathrm{H}(\mathrm{IgH})$ locus and exclude the 'stochastic' model of Cohn and Langman ${ }^{4}$. We wish to point out that their results show a remarkable agreement with the predictions of a third model, the 'cellular selection' model.

This cellular selection model postulates that there is no feedback to stop rearrangement at the IgH locus, at least not until a functional immunoglobulin molecule with both heavy and light chains is formed, and that allelic exclusion at the $\mathrm{IgH}$ locus results principally from selection against cells producing cytoplasmic heavy chain from both alleles. The toxicity of free heavy chain ${ }^{5}$ may be one factor, but by no means the only one, that could drive such cellular selection.

The cited results ${ }^{1}$ were obtained with heterozygous mice in which only one IgH allele had a disrupted membrane exon; the other allele was wild type. Cells with allelic inclusion (namely cells that produce cytoplasmic IgH chains encoded by both alleles) were enumerated. The 'expected' values calculated ${ }^{1}$, however, are not those that would be predicted using the authors' assumptions. Although this is not the principal reason for the lack of quantitative agreement, we present here the correct calculations.

Let $p_{i}$ be the probability that exactly $i$ chains are produced by a newly generated $B$ cell. The frequency of double producers is $f=p_{2} /\left(1-p_{0}\right)$, because cells producing no $\mathrm{IgH}$ chains are not scored. With the feedback model of allelic exclusion, $p_{0}=(1-h)^{2}$ and $p_{2}=h^{2} / 2$, where $h$ is the fraction of productive $V D J$ joints $(V, D$ and $J$ are, respectively, variable, diversity and joining gene segments); the factor of $1 / 2$ is needed because the mutant allele must rearrange first if both alleles are to be productively rearranged. Thus we get $f=h /(4-2 h)$ (curve $a$ in the figure). With the cellular selection model, $p_{0}=(1-h)^{2}$ and $p_{2}=h^{2}$ (a factor of $1 / 2$ is not needed because with this model it is irrelevant which allele rearranges first). Thus we get $f=h /(2-h)$ (curve $b$ in the figure).

With the feedback model, $f$ should be essentially the same for newly arising B cells in the bone marrow and for spleen 